\title{
Differential expression of apoptosis-related genes from death receptor pathway in chronic myeloproliferative diseases
}

\author{
Raquel Tognon, ${ }^{1}$ Elainy Patrícia Lino Gasparotto, ${ }^{1}$ Janine Marie Gisele Leroy, ${ }^{2}$ \\ Gislane Lelis Vilela Oliveira, ${ }^{1}$ Renata Peres Neves, ${ }^{1}$ Rita de Cássia Viu Carrara, ${ }^{3}$ \\ Simone Kashima, ${ }^{3}$ Dimas Tadeu Covas, ${ }^{3}$ Mary Santana, ${ }^{4}$ Elizabeth Xisto Souto, ${ }^{4}$ \\ Maria Aparecida Zanichelli, ${ }^{4,7}$ Carlos Eduardo Engel Velano, ${ }^{5}$ Belinda Pinto Simões, ${ }^{5}$ \\ Fernando Lopes Alberto, ${ }^{6}$ Kozue Miyashiro, ${ }^{6}$ Ana Maria de Souza, ${ }^{1}$ \\ Gustavo Pessini Amarante-Mendes, ${ }^{2}$ Fabíola Attié de Castro ${ }^{1}$
}

\begin{abstract}
- Additional tables and figures are published online only. To view these files please visit the journal online (http://jcp.bmj. com)

${ }^{1}$ Department of Clinical, Toxicological and Bromatological Analysis, University of São Paulo, Ribeirão Preto School of Pharmaceutical Sciences, Ribeirão Preto, Brazil. INCT-IF 2Department of Immunology, University of São Paulo, Institute of Biomedical Sciences, São Paulo, Brazil

${ }^{3}$ Ribeirão Preto Center for Transfusion Medicine, Hospital das Clínicas, University of São Paulo, Ribeirão Preto School of Medicine, Ribeirão Preto, Brazil ${ }^{4}$ Brigadeiro Hospital of São Paulo, São Paulo, Brazil ${ }^{5}$ Department of Clinical Medicine, University of São Paulo at Ribeirão Preto School of Medicine, Ribeirão Preto, Brazil ${ }^{6}$ Fleury Medicine and Health, São Paulo, Brazil

${ }^{7}$ Institute for cancer treatment in children-ITACI
\end{abstract}

\section{Correspondence to}

Professor Fabiola Attié de Castro, Faculdade de Ciências Farmacêuticas de Ribeirão Preto - USP, Laboratório de Hematologia, Av do Café, s/n Ribeirão Preto, SP, Brazil CEP. 14040-903;

castrofa@fcfrp.usp.br

Accepted 28 September 2010 Published Online First

2 November 2010

\section{ABSTRACT}

Background Chronic myeloproliferative disorders (MPDs) are clonal haematopoietic stem cell malignancies characterised by an accumulation of mature myeloid cells in bone marrow and peripheral blood. Deregulation of the apoptotic machinery may be associated with MPD physiopathology.

Aims To evaluate expression of death receptors' family members, mononuclear cell apoptosis resistance, and JAK2 allele burden.

Subjects and Methods Bone marrow haematopoietic progenitor CD34 cells were separated using the Ficollhypaque protocol followed by the Miltenyi CD34 isolation kit, and peripheral blood leukocytes were separated by the Haes-Steril method. Total RNA was extracted by the Trizol method, the High Capacity Kit was used to synthesise CDNA, and real-time PCR was performed using SybrGreen in ABIPrism 7500 equipment. The results of gene expression quantification are given as $2^{-\Delta \Delta \mathrm{Ct}}$. The JAK2 V617F mutation was detected by real-time allelic discrimination PCR assay. Peripheral blood mononuclear cells (PBMCs) were isolated by the Ficoll-hypaque protocol and cultured in the presence of apoptosis inducers.

Results In CD34 cells, there was mRNA overexpression for fas, faim and c-flip in polycythaemia vera (PV), essential thrombocythaemia (ET) and primary myelofibrosis (PMF), as well as fas/ in PMF, and $d r 4$ levels were increased in ET. In leukocytes, fas, c-flip and trail levels were increased in PV, and $d r 5$ expression was decreased in ET. There was an association between $d r 5$ and fas/ expression and JAK2V617F mutation. PBMCs from patients with PV, ET or PMF showed resistance to apoptosis inducers.

Conclusions The results indicate deregulation of apoptosis gene expression, which may be associated with MPD pathogenesis leading to accumulation of myeloid cells in MPDs.

\section{INTRODUCTION}

Myeloproliferative disorders (MPDs) are clonal haematopoietic stem cell malignancies characterised primarily by hypercellular bone marrow, proliferation of cells from one or several myeloid lineages, and haematopoietic progenitor independence of, or hypersensitivity to, cytokines. ${ }^{1-3}$
According to the World Health Organization (WHO), myeloproliferative neoplasms can be divided into two groups: classic MPDs, comprising chronic myelogenous leukaemia (CML), polycythaemia vera (PV), essential thrombocythaemia (ET) and primary myelofibrosis (PMF); and less common MPDs, such as chronic neutrophilic leukaemia. ${ }^{4}$

MPDs are heterogeneous in their molecular aspects and natural course, but their physiopathology often involves deregulation of tyrosine kinases, such as that of $\mathrm{Bcr}-\mathrm{Abl}$ in the pathogenesis of CML. ${ }^{15}$

In 2005, several independent groups reported in patients with MPD a somatic mutation in the Janus kinase JAK2, which has a key role in the signal transduction pathway of various haematopoietic growth factor receptors. ${ }^{2}{ }^{6-9}$ The point mutation that led to valine-to-phenylalanine substitution at polypeptide position 617 is designated JAK2V617F and is responsible for constitutive kinase activity. $^{10-14}$

JAK2V617F mutation has been reported in approximately half of all patients with PMF and ET and in nearly all of those with $\mathrm{PV}^{2}{ }^{6-8}$ 15-17 Increased JAK2V617F allele burden has been associated with upregulated expression of downstream JAK2 target genes, such as polycythaemia rubra vera 1 (PRV-1). ${ }^{616} 17$ There are also indications that patient mutation status correlates with prognosis and treatment response. ${ }^{18-21}$

Regardless of the recent advances in MPD diagnosis and description of molecular markers, clearer reports of the mechanisms involved in the physiopathology of MPDs are needed to identify new markers of diagnostic and prognostic molecular targets for therapy. In this context, we can hypothesise that deregulation of apoptosis contributes to the myeloaccumulation seen in these disorders, since there is a constant need to control the numbers of haematopoietic cells because of the great potential for intrinsic proliferation and cell turnover. $^{22-24}$ The literature data reinforce our hypothesis, since deregulation of the apoptosis process has been reported in various myeloid haematological neoplasias such as chronic neutrophilic leukaemia, ${ }^{25}$ myelodysplastic syndromes, ${ }^{26}$ 
$\mathrm{CML}^{27}$ and in MPD chromosome Ph negative. ${ }^{28} 29$ In myelodysplastic syndromes, it has been described as an altered expression of genes related to the intrinsic apoptosis pathway. ${ }^{26}$ In CML, the cells are resistant to apoptosis due to the kinase Bcr-Abl, and overexpression of anti-apoptotic genes has been described. ${ }^{27}$ In MPD chromosome $\mathrm{Ph}$ negative, little is known about apoptosisrelated gene expression. Zeuner et $a l^{28}$ in PV found c-flip overexpression, and Ciurea et a ${ }^{29}$ described a different development of megakaryocyte apoptosis and overexpression of anti-apoptotic protein $\mathrm{Bcl}-\mathrm{x}_{\mathrm{L}}$ in PMF. Laubach and collaborators ${ }^{30}$ reported that PV-deregulated erythropoiesis is linked to erythropoietin hypersensitivity and apoptosis resistance of erythroid precursor cells.

Taken together, all this information from the literature indicates that, in most cases, apoptosis deregulation is associated with haematological neoplasia pathogenesis. ${ }^{25-31}$

Two major apoptotic pathways have been well characterised: one is activated by changes in mitochondrial permeability (intrinsic pathway); the other is triggered by cell surface death receptors engaging their specific ligands (extrinsic pathway). ${ }^{32}$ Activation of the death receptor pathway, which primarily includes CD95 (APO-1/Fas) or TRAIL receptors (DR4 and DR5) and their ligands (FasL and TRAIL, respectively), likewise the anti-apoptotic molecules FAIM and C-FLIP, results in recruitment of adaptor molecules and caspase- 8 to form the death-inducing signalling complex, thereby inducing caspase-3 activation and apoptosis. $^{24} 33$

The aim of this study was to quantify expression of the genes fas, fasl, faim, c-flip, dr4, drs and trail, all of which participate in mechanisms that regulate the extrinsic pathway, in CD34 cells and leukocytes from patients with MPDs. We also attempted to detect mononuclear cell susceptibility to death stimulated by apoptosis inducers and to correlate the results with JAK2V617F allele burden.

\section{SUBJECTS AND METHODS Patients and controls}

In this study, bone marrow and peripheral blood samples from 26 patients with ET, 12 with PV, and 11 with PMF were evaluated. There were 17 men and 32 women, mean age was 61.1 years (range, 35-83), and 43 patients were white and six were black (online supplementary table 1). All of the patients selected had been diagnosed according to WHO criteria. ${ }^{4}$ The controls comprised bone marrow samples collected from 23 healthy subjects (12 male, 11 female; mean age 35.9 years (range 14-54)) and peripheral blood samples from 30 subjects (11 male, 19 female; mean age 54.5 years (range $31-80)$ ). Patients were selected from those treated at the Brigadeiro Hospital as well as from the Hospital das Clínicas of the University of São Paulo at Ribeirão Preto School of Medicine, state of São Paulo, Brazil. Healthy subjects were recruited from the University of São Paulo at Ribeirão Preto School of Pharmaceutical Sciences, whereas those who provided bone marrow samples were recruited from among marrow donors at the Bone Marrow Transplantation Unit of the Hospital das Clínicas of the University of São Paulo. There is a significant age difference between bone marrow controls and the patient population because the local ethics committees would only permit bone marrow samples to be obtained from bone marrow donors during the bone marrow transplantation cell collection procedure.

\section{Isolation of peripheral blood mononuclear cells (PBMCs) and leukocytes}

PBMCs were isolated using the Ficoll-hypaque density gradient protocol (Histopaque; Sigma-Aldrich, St Louis, Missouri, USA) according to the manufacturer's instructions. The PBMCs isolated were used to assess lymphocyte apoptosis assays.

Leukocytes were obtained from the peripheral blood of patients and controls using Haes-Steril reagent (Voluven; Frasenius Kabi, Campinas, Brazil).

\section{CD34 haematopoietic stem cell separation}

Haematopoietic progenitor CD34 cells collected from patients and controls were separated from bone marrow mononuclear cells using the MidiMacs CD34+ Isolation Kit (MACS; Miltenyi Biotec, Bergisch Gladbach, Germany) according to the manufacturer's protocol. CD34 purity was determined by flow cytometry (FACSort; Becton Dickinson, San Jose, California, USA). CD34 cells with $\geq 80 \%$ purity were used in the detection of gene expression by quantitative real-time PCR.

\section{Total RNA extraction, cDNA synthesis and real-time PCR}

Total RNA was extracted from leukocytes and CD34 cells using Trizol methods (Invitrogen Life Technologies, Carlsbad, California, USA) as described by the manufacturer. RNA concentration and purity was determined spectrophotometrically by measuring fluorescence at $260 \mathrm{~nm}$ and $280 \mathrm{~nm}$. RNA (1 $\mu \mathrm{g})$ was reversetranscribed to cDNA using the High Capacity cDNA Archive Kit (Applied Biosystems, Foster City, California, USA), also according to the manufacturer's instructions. cDNA ( $1 \mu \mathrm{l}$ of cDNA diluted 1:3) was applied in the real-time PCR assay to determine the expression of c-flip, dr4, dr5, fas, fasl, faim and trail. Gene expression was quantified using the SYBR Green PCR Master Mix Kit (Applied Biosystems) and specific primers (Invitrogen Life Technologies) (online supplementary table 2) on the 7500 Real Time PCR System (Applied Biosystems). Real-time PCR conditions were: $50^{\circ} \mathrm{C}$ for $2 \mathrm{~min} ; 95^{\circ} \mathrm{C}$ for $10 \mathrm{~min}$; and 50 cycles at $95^{\circ} \mathrm{C}$ for $15 \mathrm{~s} ; 54$ at $60^{\circ} \mathrm{C}$ (specific for target gene) for $25 \mathrm{~s}$; and $72^{\circ} \mathrm{C}$ for $34 \mathrm{~s}$. The annealing temperatures of each set of primers are also shown in supplementary table 1 . $\beta$-actin and GAPDH genes were used as endogenous controls (housekeeping genes). The amount of mRNA for each sample was normalised using the geometric average of the housekeeping genes. All reactions were duplicated, and the relative expression levels were calculated using the $2^{-\Delta \Delta \mathrm{Ct}}$ method $^{34}$ with the mean of the control group used as calibrator.

\section{Detection of JAK2V617F mutation and determination of JAK2V617 allele burden}

The JAK2V617F mutation was detected in a reference laboratory (Fleury Medicine and Health, São Paulo, SP, Brazil) by real-time allelic discrimination PCR assay. The assay used two fluorogenic, minor groove binding probes to detect the $\mathrm{G} \rightarrow \mathrm{T}$ mutation in exon 14 of JAK2 gene. Sequences of primers (forward primer, 5'-GCAGCAAGTATGATGAGCAAGCT-3'; reverse primer, 5'-GGCATTAGAAAGCCTGTAGTTTTACTTAC-3') and minor groove binding probes were designed by using Primer Express software, version 1.5 (Applied Biosystems). The hybridisation probes were designed with different dyes to allow single-tube allelic discrimination. One probe with the reporter FAM (6-carboxyfluorescein) was targeted to the wild-type allele (5'-6FAM-TGGAGTATGTGTCTGTGGA-3') and the other probe with the reporter VIC (6-carboxyrhodamine 6G) was targeted to the mutant allele (5'-VIC-TGGAGTATGTTTCTGTGGAG-3'). Primers were synthesised by IDT (Integrated DNA Technologies, Coralville, Iowa, USA) and probes were synthesised by Applied Biosystems. Amplification by real-time PCR was performed using TaqMan Universal Master Mix (Applied Biosystems). Thermal cycling conditions consisted of $50^{\circ} \mathrm{C}$ for $2 \mathrm{~min}, 45$ 
cycles of $95^{\circ} \mathrm{C}$ for $15 \mathrm{~s}$ and $60^{\circ} \mathrm{C}$ for $1 \mathrm{~min}$ on the Rotor-Gene 3000 (Corbett Research, Sydney, Australia). Each PCR assay included a control without added DNA (no-template control).

The allele burden was estimated based on the normalised fluorescent signal of the mutant allele and wild-type allele of real-time PCR and reported as a percentage of JAK2V617F mutation or negative.

\section{Assessment of mononuclear cell apoptosis}

Mononuclear cells were cultured in complete RPMI 1640 medium (Sigma) supplemented with $10 \mathrm{mM}$ Hepes, $200 \mathrm{mM}$ L-glutamine, 100 units $/ \mathrm{ml}$ penicillin, $100 \mu \mathrm{g} / \mathrm{ml}$ streptomycin and $10 \%$ fetal bovine serum at $37^{\circ} \mathrm{C}$ in an atmosphere of $5 \%$ $\mathrm{CO}_{2}$ for $12 \mathrm{~h}$ in the presence of apoptosis inducers: actinomycin (ACT-D, $1 \mu \mathrm{M}$; Sigma); etoposide (VP16, $25 \mu \mathrm{M}$; Bristol-MeyersSquibb, Mayaguez, Puerto Rico); teniposide (VM26, $25 \mu \mathrm{M}$; Bristol-Meyers-Squibb); cytarabine (ARA-C, $25 \mu \mathrm{M}$; Pfizer, Milano, Italy); staurosporine (STS, $0.5 \mu \mathrm{M}$; Sigma-Aldrich).

Cell apoptosis was quantified by flow cytometry, using the annexin-V/fluorescein isothiocyanate (FITC) technique. Mononuclear cells were recovered from culture medium by centrifugation at $240 \times \mathrm{g}$ for $10 \mathrm{~min}$ at $4^{\circ} \mathrm{C}$, washed once with annexin buffer (10 mM Hepes, pH 7.4; $150 \mathrm{mM} \mathrm{NaCl} ; 5 \mathrm{mM}$ $\mathrm{KCl} ; 1 \mathrm{mM} \mathrm{MgCl}_{2} ; 1.8 \mathrm{mM} \mathrm{CaCl}_{2}$ ) and incubated for $20 \mathrm{~min}$ in the dark with $100 \mu \mathrm{l}$ annexin-V/FITC. Subsequently, $40 \mu \mathrm{l}$ propidium iodide (PI) solution $(50 \mu \mathrm{g} / \mathrm{ml})$ was added to each tube, and the contents were analysed by flow cytometry. Five thousand mononuclear cells were acquired using a FACS Canto Flow Cytometer (Becton-Dickinson) and analysed by dot-plot using Diva 6.0 Software (Becton-Dickinson). The results are given as a percentage of the apoptotic cells (mononuclear cells positive for annexin-V and PI negative staining). A representative FACS plot of annexin/PI staining showing the full gating strategy on mononuclear cells (forward scatter and side scatter gate) and the dot-plot analysis of negative control and mononuclear cells stimulated by teniposide (VM26) are shown in figure $1 \mathrm{~A}-\mathrm{C}$.

Although it is controversial, many authors ${ }^{35-38}$ have reported lymphoid lineage involvement in PV, ET and PMF. Thus, we chose mononuclear cells to perform the assays of apoptosis measurement because the mononuclear cell isolation protocol produces a cell population with high purity, and FACS analysis well discriminates between lymphocytes and monocytes stimulated by apoptotic inducers.

\section{Statistical analysis}

Data related to gene expression and percentages of apoptotic cells from patients and controls were compared by the non-parametric Mann-Whitney test. To draw correlations between JAK2V617F allele burden and gene expression, we used the non-parametric Spearman test. Statistical analyses were carried out using Prism Software V5.0. $\mathrm{p} \leq 0.05$ was considered significant.

\section{RESULTS}

fas, fasl, dr4, faim and c-flip expression is elevated in CD34 cells from patients with MPDs

As can be seen in figure 2A, fas expression was significantly higher in the PV, ET and PMF groups than in the control group $(\mathrm{p}<0.0001, \mathrm{p}=0.0013$ and $\mathrm{p}=0.0004$, respectively), and there was significant difference between the control group and PMF group in terms of CD34 fasl mRNA levels ( $p=0.0002$, figure $2 B$ ). The levels of trail and $d r 5$ in CD34 cells were comparable between patients and controls $(p>0.05)$ (online supplementary figure $1 \mathrm{~A}, \mathrm{~B})$. In contrast, dr4 expression was increased in the ET group ( $p=0.0079$; figure $2 C$ ).

Expression of the anti-apoptotic gene faim was also elevated in the three groups in comparison with the control group $(p=0.0048, p=0.0002, p=0.0076)$, as shown in figure $2 D$, and c-flip expression was also elevated in the PV, ET and PMF groups in comparison with the control group $(\mathrm{p}=0.0228, \mathrm{p}=0.0121$ and $\mathrm{p}=0.0026$, respectively; figure $2 \mathrm{E}$ ).

Analysing gene expression among MPD groups, we found a significant difference between the ET and PMF groups in terms of fasl expression ( $\mathrm{p}=0.0012$, figure $2 \mathrm{~B}$ ).

It is worth emphasising here that there is no difference in apoptosis-related gene expression $(p>0.05)$ with age (range 14-54 years, median 37.0) within the normal population (bone marrow donors) (data not shown).

\section{Expression of fas, trail, dr5, c-flip and faim is deregulated in leukocytes of patients with MPDs}

Expression of fas was higher in the PV group than in the control group ( $p=0.0013$; figure $3 \mathrm{~A})$, and fasl expression was not
A

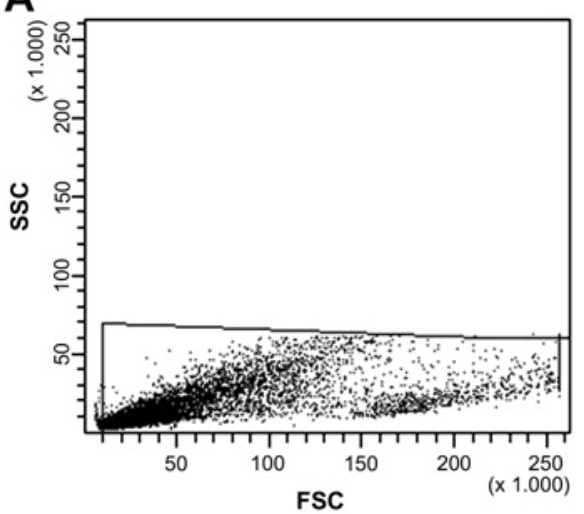

B

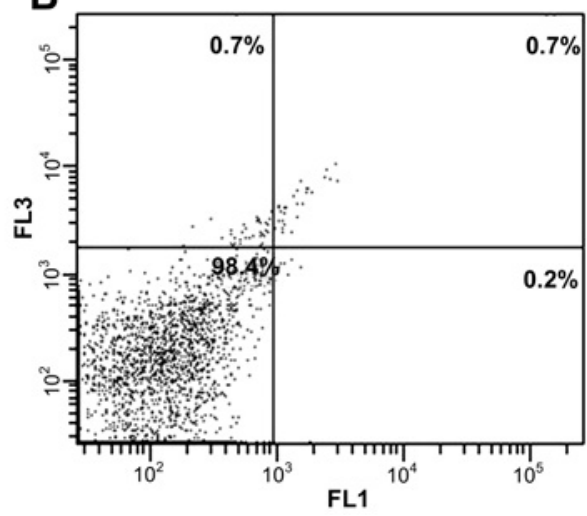

C

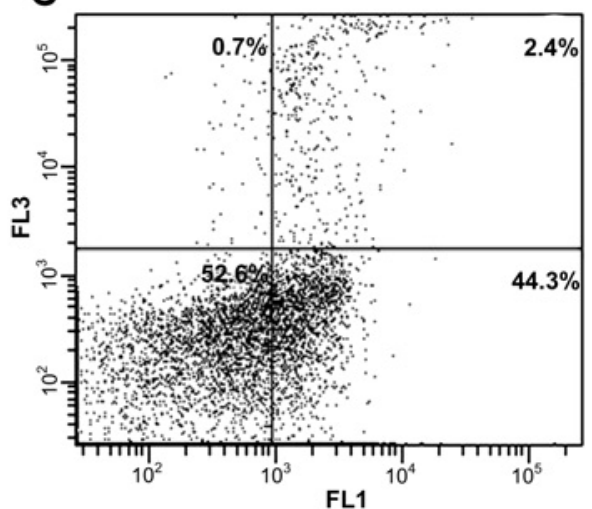

Figure 1 (A) Dot-plot diagram of gate in control mononuclear cells; (B) dot-plot diagram of fluorescein isothiocyanate (FITC)/annexin-V/propidium iodide (PI) flow cytometry of control mononuclear cells without treatment; (C) dot-plot diagram of FITC/annexin-V/PI flow cytometry of control mononuclear cells treated with $25 \mu \mathrm{M}$ teniposide. In (B) and (C), the lower right quadrants show the viable cells, which exclude PI and are negative for FITC/annexin-V binding. The upper right quadrants contain the non-viable cells, necrotic cells, positive for FITC/annexin binding and for PI uptake. The lower right quadrants represent apoptotic cells, FITC/annexin-V positive and PI negative. FSC: Forward scatter; SSC: side scatter; FL1: fluorescence channel 1; FL3: fluorescence channel 3. 

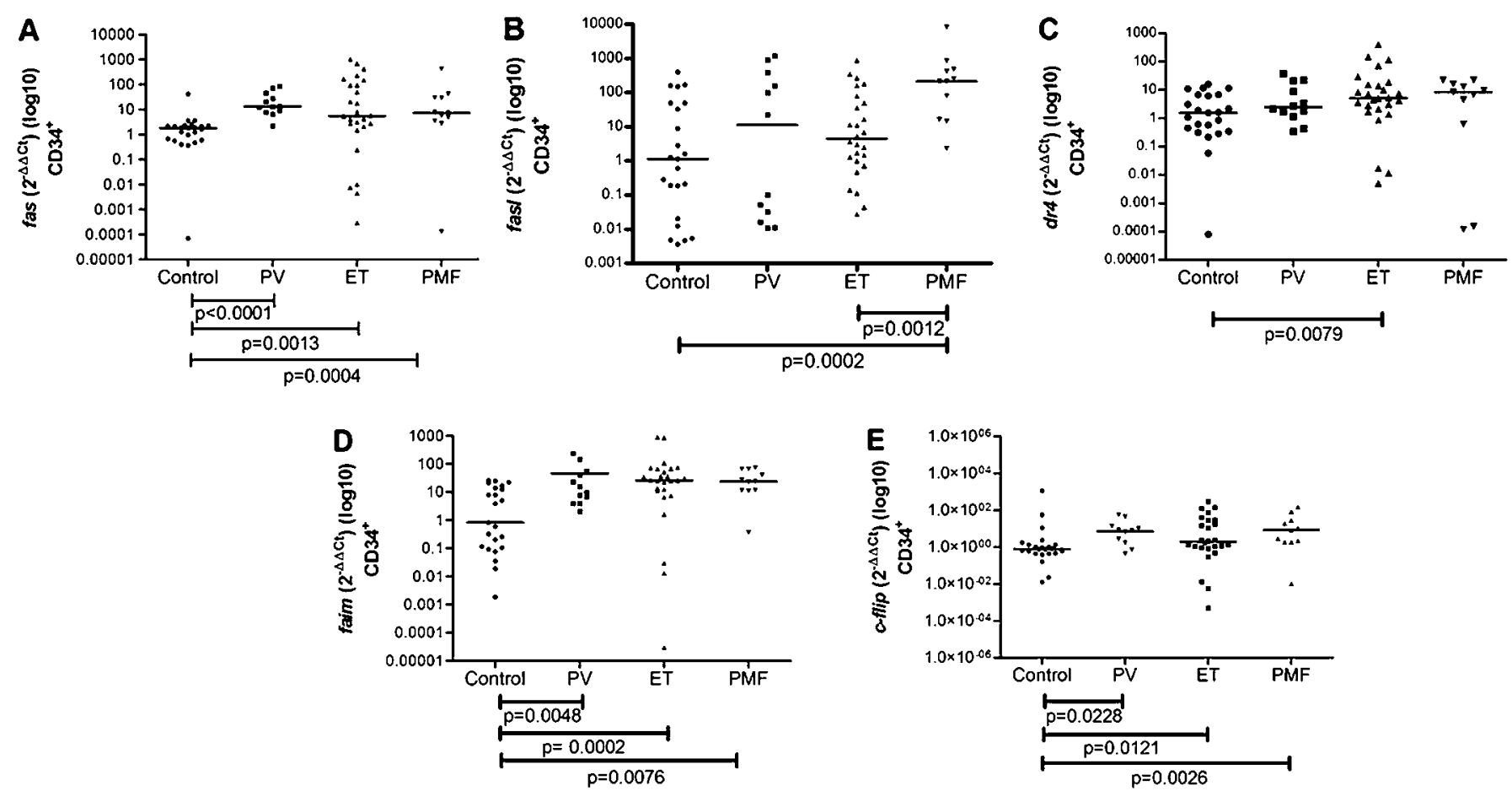

Figure 2 Gene expression in bone marrow CD34 cells of control, polycythaemia vera (PV), essential thrombocythaemia (ET) and primary myelofibrosis (PMF) groups: (A) fas (median: control, 1.80; PV, 13.16; ET, 5.52; PMF, 7.21); (B) fasl (median: control, 1.13; PV, 11.16; ET, 4.46; PMF, 213.1); (C) dr4 (median: control, 1.54; PV, 2.49; ET, 5.20; PMF, 8.43); (D) faim (median: control, 0.82; PV, 12.95; ET, 26.03; PMF, 23.56); (E) c-flip (median: control, 0.78; PV, 7.22; ET, 1.93; PMF, 8.37). All differences are $\mathrm{p}>0.05$ unless otherwise noted.

significantly different between MPD groups and the control group ( $p>0.05$ ) (online supplementary figure 1C). In addition, we found that trail mRNA concentration was higher in PV leukocytes than control group leukocytes ( $p=0.0321$, figure $3 B$ ), and $d r 5$ expression was lower in ET leukocytes ( $p=0.0387$, figure $3 C)$. dr4 expression was not significantly different between MPD groups and the control group ( $p>0.05)$ (online supplementary figure 1D).

Expression of the anti-apoptotic genes faim and c-flip was increased in the PMF $(p=0.0040)$ and PV $(p=0.0043)$ group, respectively (figure $3 \mathrm{D}, \mathrm{E}$ ).
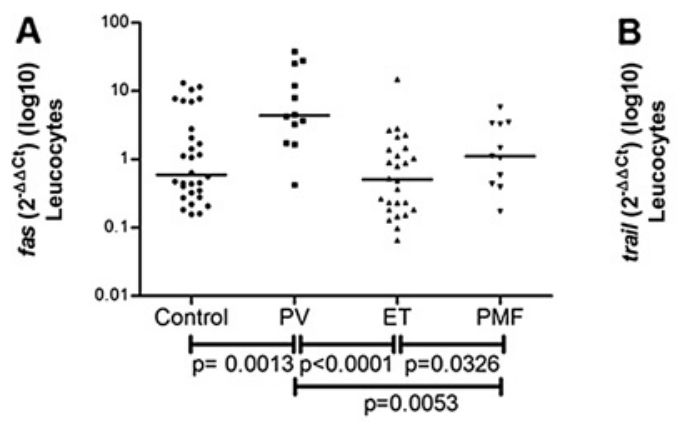
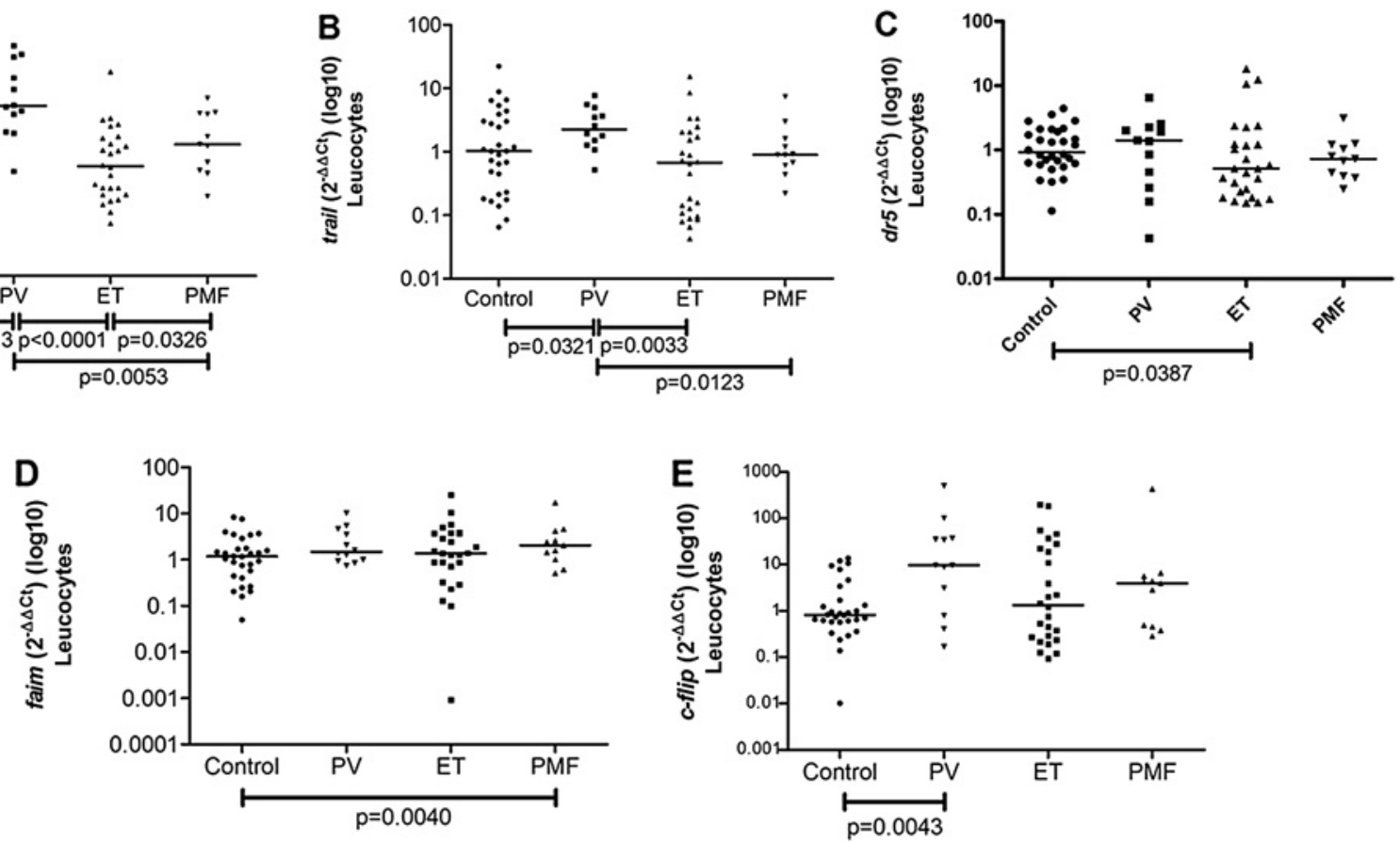

Figure 3 Gene expression in peripheral leukocytes of control, polycythaemia vera (PV), essential thrombocythaemia (ET) and primary myelofibrosis (PMF) groups: (A) fas (median: control, 0.60; PV, 4.38; ET, 0.51; PMF, 1.11); (B) trail (median: control, 1.024; PV, 2.25; ET, 0.67; PMF, 0.89); (C) dr5 (median: control, 0.92; PV, 1.40; ET, 0.52; PMF, 0.77); (D) faim (median: control, 1.18; PV, 1.49; ET, 1.37; PMF, 2.05); (E) c-flip (median: control, 0.81; PV, 9.71; ET, 1.32; PMF, 3.97). All differences are $p>0.05$ unless otherwise noted. 
Figure 4 Correlation of (A) fas/ and (B) $d r 5$ expression in polycythaemia vera (PV) patients with the JAK2V617F allele burden.
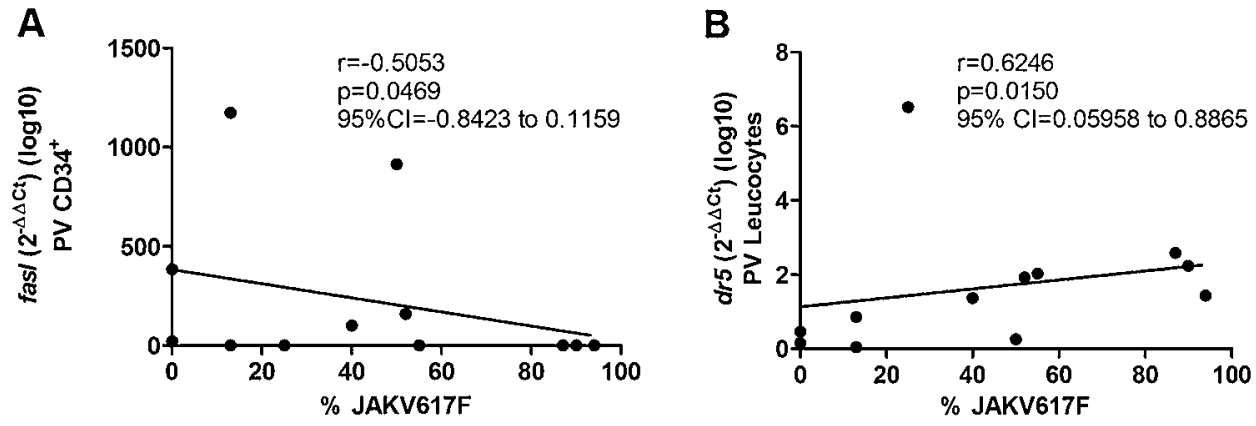

Analysing MPD leucocyte gene expression, we found a significant difference between PV and ET groups in terms of fas expression $(p<0.0001$, figure $3 A)$ and trail $(p=0.0033$, figure $3 B)$ and between PV and PMF groups in terms of fas and trail expression ( $p=0.0053$ and $p=0.0123$, respectively, figure $3 A, B$ ). We also detected that fas expression was elevated in PMF patients in comparison to ET patients ( $p=0.0326$, figure $3 \mathrm{~A})$.

\section{Correlation between gene expression and JAK2V617F mutation}

There was a correlation between the JAK2V617F allele burden and fasl expression $(\mathrm{r}=-0.5053 ; \mathrm{p}=0.0469 ; 95 \% \mathrm{CI}=-0.8423$ to 0.1159 ) in CD34 and between JAK2V617F allele burden and $d r 5$ expression ( $r=0.6246 ; p=0.0150 ; 95 \% \mathrm{CI}=0.05985$ to 0.8865$)$ in leukocytes from PV patients (figure $4 \mathrm{~A}, \mathrm{~B}$, respectively).

There was no association between apoptosis-related gene expression and allele burden in ET and PMF patients. No differences were observed in level of apoptosis-related gene expression between JAK2V617F positive and negative ET and PMF patients.

\section{Mononuclear cells of MPD patients are resistant to apoptosis inducers}

Mononuclear cell resistance to $1 \mu \mathrm{M}$ ACT-D and $25 \mu \mathrm{M}$ VP16 was higher in the PV $(p=0.03, p=0.03$, respectively), ET $(\mathrm{p}<0.0001, \mathrm{z}=0.01$, respectively) and $\mathrm{PMF}(\mathrm{p}<0.0001, \mathrm{p}<0.0001$, respectively) groups than in the control group (figure $5 \mathrm{~A}, \mathrm{~B}$ ). Cells obtained from ET and PMF patients were more resistant to apoptosis induced by $25 \mu \mathrm{M}$ VM26 than were those obtained from controls ( $p=0.005$ and $p=0.001$, respectively; figure $5 C$ ). In comparison with the values obtained for controls, the resistance of apoptotic cells to $25 \mu \mathrm{M}$ ARA-C was higher in PV, ET and PMF group cells $(p=0.031, p=0.033$ and $p=0.013$, respectively; figure $5 \mathrm{D}$ ), as was that induced by $5 \mu \mathrm{M}$ STS in ET and PMF group cells ( $p=0.0002$ and $p<0.0001$, respectively, figure $5 E$ ).
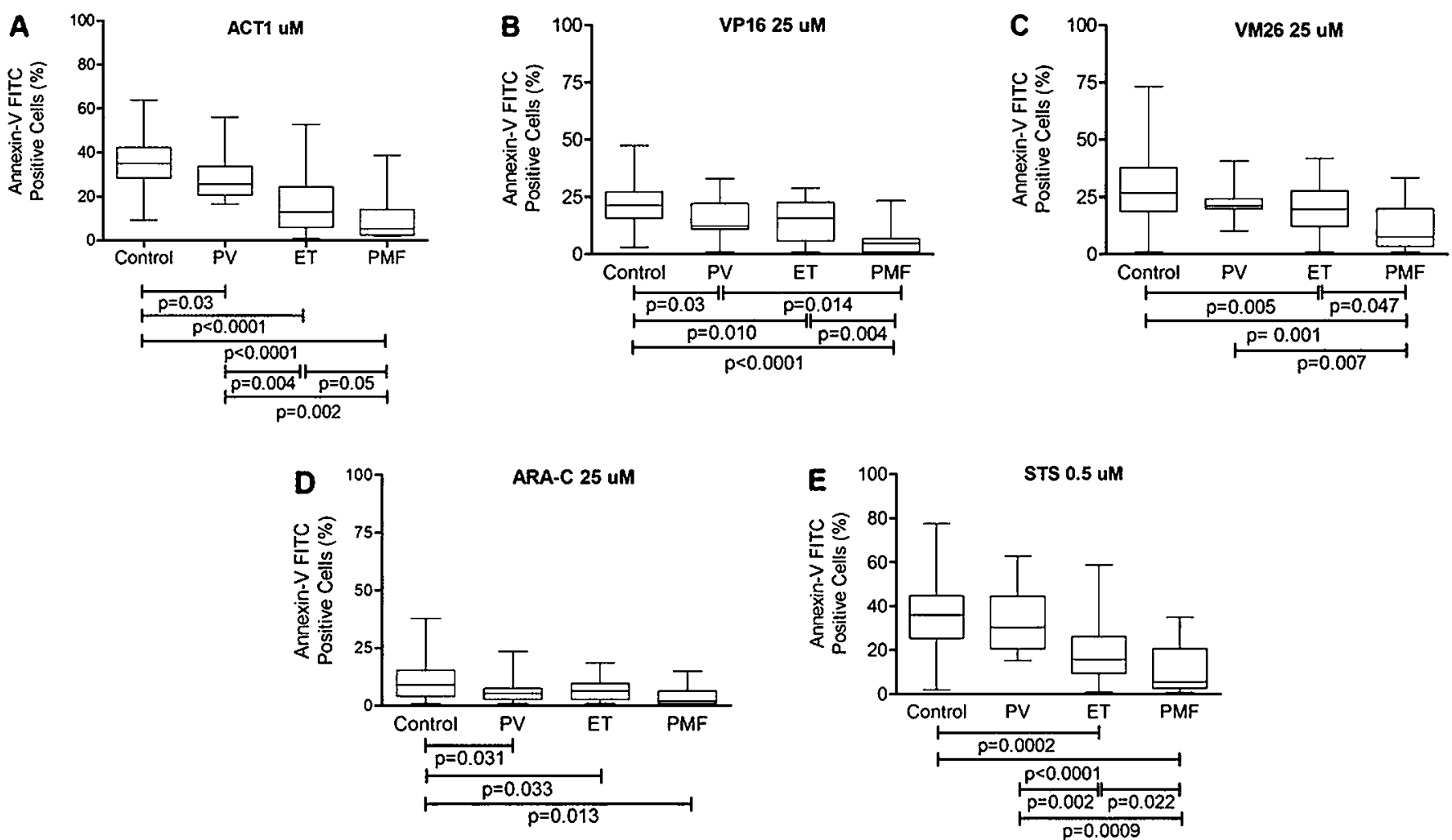

Figure 5 Percentage of mononuclear cell apoptosis induced by drugs and detected by annexin-V/fluorescein isothiocyanate (FITC) in control, polycythaemia vera (PV), essential thrombocythaemia (ET) and primary myelofibrosis (PMF) groups: (A) $1 \mu \mathrm{M}$ ACT-D (median: control, 35.10; PV, 25.50; ET, 13.00; PMF, 5.25); (B) $25 \mu \mathrm{M}$ VP16 (median: control, 21.41; PV, 12.25; ET, 15.70; PMF, 4.90); (C) $25 \mu \mathrm{M}$ VM26 (median: control, 26.80 ; PV, 21.00; ET, 19.60; PMF, 7.70); (D) $25 \mu \mathrm{M}$ ARA-C (median: control, 9.10; PV, 5.40; ET, 6.35; PMF, 1.90); (E) 0.5 $\mu$ M STS (median: control, 36.05 ; PV, 30.35; ET, 15.80; PMF, 5.50). All differences are $p>0.05$ unless otherwise noted. The box and whisker graphs show the maximum, median and minimum of the mononuclear cell apoptosis percentage. 
Among MPD groups, we detected a difference in mononuclear cell susceptibility to: $1 \mu \mathrm{M}$ ACT comparing PV and ET groups $(p=0.004)$, PV and PMF groups $(p=0.002)$ and ET and PMF groups $(p=0.05)$ (figure $5 \mathrm{~A}) ; 25 \mu \mathrm{M}$ VP16 between $\mathrm{PV}$ and PMF groups $(p=0.014)$ and between ET and PMF groups $(\mathrm{p}=0.004)$ (figure $5 \mathrm{~B}) ; 25 \mu \mathrm{M} \mathrm{VM} 26$ comparing $\mathrm{PV}$ and $\mathrm{PMF}$ groups $(p=0.007)$ and comparing ET and PMF groups $(p=0.047)$ (figure 5C); and $0.5 \mu \mathrm{M}$ STS between PV and ET groups $(p=0.002)$, between PV and PMF groups $(p=0.0009)$ and between ET and PMF groups $(p=0.022)$ (figure $5 E$ ).

\section{DISCUSSION}

MPDs are characterised by increased numbers of myeloid cells, a lineage that typically predominates. The mechanisms whereby myeloproliferation, disease progression and development occur in MPDs remain unknown. Abnormal expression of apoptosis regulatory genes has been described in oncological and haematological diseases, and associations between regulatory gene expression and the pathogenesis of such diseases have been demonstrated. ${ }^{26} 2839$

Thus, we investigated here the expression of the genes fas, fasl, faim, c-flip, dr4, dr5 and trail in CD34 haematopoietic stem cells and leukocytes from patients with MPDs. We detected a potential correlation between apoptosis-related gene expression with JAK2V617F mutation and mononuclear cell apoptosis.

It is noteworthy that we found alterations in Fas systemrelated gene expression in CD34 cells and leukocytes from patients with MPDs. It is well known that the Fas/FasL system is critically involved in haematopoietic cell survival and apoptosis. $^{24} 3340$ Under physiological conditions, CD34 bone marrow cells express Fas at lower levels; during haematopoietic cell differentiation, the receptor is expressed in proliferating and differentiating progenitors, serving as a negative regulator of distal differentiation in all lineages. ${ }^{41-43}$ FAIM, the expression of which is also deregulated in MPD patients evaluated here, is a Fas antagonist that has been reported to be upregulated in resistant $B$ cells and functions as an inhibitor of Fas-induced cell death. FAIM is highly conserved in evolution and is broadly expressed in many tissues. ${ }^{44-47}$
Regarding c-flip expression, our data corroborate data in the literature on PV and ET. ${ }^{28} 48$ Zeuner et al ${ }^{28}$ showed an increase in FLIPshort expression in PV erythroid precursor cells. These authors found that JAK2V617F mutation in PV erythroblasts obtained in vitro was associated with cytokine-independent activation of the ERK/MAPK and PI3K pathways and deregulated expression of c-flip. They also showed that one patient in three with wild-type JAK2 was resistant to death receptor activation and had extremely high levels of c-flip, indicating that c-flip overexpression may also contribute to death receptor resistance in PV patients with wild-type JAK2. ${ }^{28}$

Tenedini et $a l^{48}$ described overexpression of c-flip in a global gene expression study of normal and malignant megakaryocytic cells from ET patients. They concluded that there is an imbalance between proliferation and apoptosis in malignant ET megakaryocytopoiesis. ${ }^{48}$ We also evaluated the ligand TRAIL, a member of the tumour necrosis factor superfamily of genes, and expression of its two death receptors, DR4 and DR5. The true physiological role of the TRAIL molecule remains unclear. However, various studies have shown that it is a potent inductor of apoptosis in a wide range of cancer cell lines, although not in many normal cells. ${ }^{30} 49-51$

In the present study, we did not find any alteration in trail expression in CD34 cells, but it was increased in leukocytes from MPD patients. Deregulated expression of death receptors dr4 and $d r 5$ in CD34 cells and leukocytes from ET was also observed. Despite the fact that there are few data on apoptotic gene expression in MPD patients, these findings are in agreement with our hypothesis that apoptosis is deregulated in Bcr-Ablnegative patients with MPD. Besides, these molecules may be used in the future as molecular markers or prognostic factors for these diseases, since $d r 4$ expression has been shown to correlate positively with tumour grade in patients with breast cancer who have invasive ductal carcinoma, ${ }^{52}$ and it has been shown that there is a significant association between high $d r 5$ expression and reduced overall survival. ${ }^{53}$ Furthermore, TRAIL therapy has been used in the treatment of neoplasia, and resistance to this treatment due to mechanisms such as changes in the expression of the anti-apoptotic proteins c-flip and $m c l-1^{54}$ has been demonstrated. ${ }^{556}$

Figure 6 Adaptation of extrinsic apoptosis pathway showing alterations in gene expression in CD34 cells and leukocytes from polycythaemia vera (PV; filled arrows), essential thrombocythaemia (ET; cross-hatched arrows) and primary myelofibrosis (PMF; discontinuous arrows). CASP, caspase. 
It is well known that the clinical course is less favourable among JAK2V617F-positive patients, and a high percentage of JAK2V617F mutations have been found to be associated with proliferation of clonogenic progenitors independently of exogenous cytokine stimulation. ${ }^{57}$ It has been speculated that constitutive activation of JAK2 correlates with changes in gene expression, since JAK2V617F activates the ERK/MAPK and PI3K pathways, as well as the STAT5 pathway, resulting in sustained phosphorylation of these molecules and cell growth in the absence of the appropriate stimulus. ${ }^{2}$ This would also protect cells from death receptor-induced apoptosis through multiple mechanisms, including 'upmodulation' of c-flip. $.^{28} 58-60$ Our results suggest a relation between this molecular abnormality and fasl and dr5 expression in PV patients. However, as we analysed seven genes and three diseases in this study, and the significance of the correlation of JAK2V617F allele burden and fasl expression was weak, we cannot confirm that the deregulated expression of the apoptosis-related genes analysed in this study is linked to JAK2V617F mutation.

We also assessed mononuclear cell apoptosis in MPD patients by using apoptosis inducers. The results suggest that the mononuclear cells of MPD patients were resistant to most of the drugs used in the assay, whereas those of the control group were not. The apoptosis inducers used in this study had different mechanisms of action for activating the intrinsic and extrinsic apoptosis pathways. For example, ACT-D inhibits DNA synthesis, triggering the intrinsic pathway, and also decreases expression of the anti-apoptotic gene c-flip, as well as amplifying fasl and TRAIL-mediated apoptosis. ${ }^{61}$ The percentage of apoptosis was lowest in patients with PMF, indicating that the level of resistance was highest in those patients.

c-flip overexpression in MPDs may block the apoptosis process by inhibiting procaspase- 8 activation at the death-inducing signalling complexes by blocking its processing. Inhibition of caspase-8 cleavage leads to impairment of the activation of downstream effector caspases, hampering propagation of the apoptotic signal. faim upregulation also contributes to cell resistance to apoptosis in MPDs, since this molecule is a mediator of Fas resistance. FAIM plays a role in modulating Fasmediated apoptosis and may act by influencing expression of c-FLIP and regulating the physical binding of caspase- 8 to Fas. ${ }^{62}$ Deregulation of fasl, fas and dr4 expression in the MPD patients evaluated may have also interfered with cell sensitivity to apoptosis; however, faim and c-flip negatively regulated activation of apoptosis pathway downstream receptors.

Taken together, our results indicate that MPD results in deregulation of the expression of genes that regulate the extrinsic pathway of the apoptotic mechanism (figure 6). To our knowledge, this is the first study to simultaneously evaluate the

\section{Take-home messages}

- In myeloproliferative diseases (MPDs) there is deregulation of expression of apoptosis-related genes from the extrinsic pathway (fas, fasl, faim, c-flip, trail, dr4 and dr5).

- Deregulation of expression of apoptosis-related genes from the extrinsic pathway may contribute to the MPD pathogenesis, leading to cell apoptosis resistance.

- fasI and $d r 5$ expression correlate with JAK2V617F mutation in patients with polycythaemia vera. expression of these specific anti- and pro-apoptotic death receptor pathway genes in Philadelphia-negative MPD patients, associating the results with apoptosis function and JAK2 allele burden. The data obtained in this investigation support the idea that deregulation of apoptosis may contribute, at least in part, to MPD pathogenesis.

The detection and monitoring of apoptosis-related gene expression as a biomarker may help in future identification of attractive candidates for new targeted monotherapies or combined therapies for MPD treatment.

Acknowledgements We thank Marcella Grando and Zita Gregório for their technical assistance and help in collecting peripheral blood samples. We are also grateful to Amélia Regina de Albuquerque for help with creating the figures, and Fabiana Rosseto de Morais and Patrícia V. B. Palma for assistance with flow cytometry assays.

Funding $\mathrm{RT}$ is the recipient of a fellowship from the Fundação de Amparo à Pesquisa do Estado de São Paulo (FAPESP) (No 08/54387-5), EPLG and RPN are recipients of fellowships from the Conselho Nacional de Desenvolvimento Científico e Tecnológico (CNPq), and GLVO is the recipient of a fellowship from the Coordenação de Aperfeiçoamento de Pessoal de Nível Superior (CAPES). This work was supported by a FAPESP grant (No 06/50094-8).

Ethics approval This study was conducted with the approval of the Brigadeiro Hospital and Hospital das Clínicas of the University of São Paulo at Ribeirão Preto School of Medicine ethics committees (No 014/06 and No 10529/2006, respectively).

Contributors RT designed and performed experiments, analysed data and wrote the paper. EPLG, GLVO, JMG, RPN, RCVC, AMS and SK performed some of the cell isolation and RNA extraction experiments. MAZ, EXS, DTC, CEEV, BPS and MS selected and treated the patients included in this study, as well as collecting the bone marrow samples for CD34+ cell isolation. MAZ, DTC, GPAM, AMS and SK discussed and revised the paper. FAC conceived the project, created the study design, analysed data, procured funding and wrote the paper.

Provenance and peer review Not commissioned; externally peer reviewed.

\section{REFERENCES}

1. Delhommeau $\mathbf{F}$, Pisani D, James $C$, et al. Oncogenic mechanisms in myeloproliferative disorders. Cell Mol Life Sci 2006:63:2939-53.

2. James C, Ugo V, Le Couedic JP, et al. A unique clonal JAK2 mutation leading to constitutive signalling causes polycythaemia vera. Nature 2005:434:1144-8.

3. Spivak JL. The chronic myeloproliferative disorders: clonality and clinical heterogeneity. Semin Hematol 2004;41(2 Suppl 3):1-5.

4. Teferri A, Vardimann JW. Classification and diagnosis of myeloproliferative neoplasms: the 2008 World Health Organization criteria and point-of-care diagnostic algorithms. Leukemia 2008;22:14-22.

5. Reilly JT. Idiopathic myelofibrosis: pathogenesis to treatment. Hematol Oncol 2006;24:56-63.

6. Kralovics R, Passamonti F, Buser AS, et al. A gain-of-function mutation of JAK2 in myeloproliferative disorders. N Engl J Med 2005;352:1779-90.

7. Baxter EJ, Scott LM, Campbell PJ, et al. Acquired mutation of the tyrosine kinase JAK2 in human myeloproliferative disorders. Lancet 2005;365:1054-61.

8. Levine RL, Wadleigh $\mathrm{M}$, Cools $\mathrm{J}$, et al. Activating mutation in the tyrosine kinase JAK2 in polycythemia vera, essential thrombocythemia, and myeloid metaplasia with myelofibrosis. Cancer Cell 2005; 7:387-97.

9. Zhao R, Xing S, Li Z, et al. Identification of an acquired JAK2 mutation in polycythemia vera. J Biol Chem 2005;280:22788-92.

10. Speletas M, Katodritou E, Daiou C, et al. Correlations of JAK2-V617F mutation with clinical and laboratory findings in patients with myeloproliferative disorders. Leuk Res 2007;31:1053-62.

11. Levine RL, Pardanani A, Tefferi $A$, et al. Role of JAK2 in the pathogenesis and therapy of myeloproliferative disorders. Nat Rev Cancer 2007;7:673-83.

12. Jones A, Kreil S, Zol K, et al. Widespread occurrence of JAK2- V617F mutation in chronic myeloproliferative disorders. Blood 2005;106:2162-68.

13. Ugo V, Marzac C, Teyssandier I, et al. Multiple signalling pathways are involved in erythropoietin-independent differentiation of erythroid progenitors in polycythemia vera. Exp Hematol 2004;32:179-87.

14. Komura E, Chagraoui H, Mansat de Mas V, et al. Spontaneous STAT5 activation induces growth factor independence in idiopathic myelofibrosis: possible relationship with FKBP51 overexpression. Exp Hematol 2003;31:622-30.

15. Barosi G, Bergamaschi G, Marchetti M, et al. JAK2 V617F mutational status predicts progression to large splenomegaly and leukemic transformation in primary myelofibrosis. Blood 2007;110:4030-6.

16. Lippert $\mathbf{E}$, Boissinot $M$, Kralovics $\mathrm{R}$, et al. The JAK2-V617F mutation is frequently present at diagnosis in patients with essential thrombocythemia and polycythemia vera. Blood 2006;108:1865-7. 
17. Larsen TS, Pallisgaard N, Møller MB, et al. The JAK2 V617F allele burden in essential thrombocythemia, polycythemia vera and primary myelofibrosis-impact on disease phenotype. Eur J Haematol 2007;79:508-15.

18. Campbell PJ, Griesshammer M, Dohner K, et al. The V617F mutation in JAK2 is associated with poorer survival in idiopathic myelofibrosis. Blood 2006;107:2098-100.

19. Sirhan S, Lasho TL, Hanson CA, et al. The presence of JAK2V617F in primary myelofibrosis or its allele burden in polycythemia vera predicts chemosensitivity to hydroxyurea. Am J Hematol 2008:83:363-5.

20. Kralovics R, Buser AS, Teo SS, et al. Comparison of molecular markers in a cohort of patients with chronic myeloproliferative disorders. Blood 2003;102: 1869-71.

21. Mesa RA, Tefferi A, Lasho TS, et al. Janus kinase 2 (V617F) mutation status, signal transducer and activator of transcription-3 phosphorylation and impaired neutrophil apoptosis in myelofibrosis with myeloid metaplasia. Leukemia 2006;20:1800-8.

22. Wickremasinghe RG, Hoffbrand AV. Biochemical and genetic control of apoptosis: relevance to normal hematopoiesis and haematological malignancies. Blood 1999;93:3587-600.

23. Schimmer AD, Hedley DW, Penn LZ, et al. Receptor and mitochondrial-mediated apoptosis in acute leukemia: a translational view. Blood 2001;98:3541-53.

24. Debatin KM, Stahnke K, Fulda S. Apoptosis in haematological disorders. Semin Cancer Biol 2003:13:149-58.

25. Hara $\mathbf{K}$, Abe $\mathrm{Y}$, Hirase $\mathrm{N}$, et al. Apoptosis resistance of mature neutrophils in a case of chronic neutrophilic leukaemia. Eur J Haematol 2001;66:70-1.

26. Economopoulou C, Pappa V, Kontsioti F, et al. Analysis of apoptosis regulatory genes expression in the bone marrow (BM) of adult de novo myelodysplastic syndromes (MDS). Leuk Res 2008;32:61-9.

27. Castro FA, Jacysyn JF, Ulbrich AG, et al. Overexpression of the anti-apoptotic genes mcl-1, bcl-w, bcl-xL and a1 is correlated with to Chronic Myelogenous Leukemia progression and resistance to Gleevec [ASH Annual Meeting Abstract]. Blood 2005; 106:P2880

28. Zeuner A, Pedini F, Signore M, et al. Increased death receptor resistance and FLIP short expression in polycythemia vera erythroid precursor cells. Blood 2006;107:3495-502

29. Ciurea S0, Merchant D, Mahmud N. Pivotal contributions of megakaryocytes to the biology of idiopathic myelofibrosis. Blood 2007;110:986-93.

30. Laubach JP, Fu P, Jiang $X$, et al. Polycythemia vera erythroid precursors exhibit increased proliferation and apoptosis resistance associated with abnormal RAS and PI3K pathway activation. Exp Hematol 2009;37:1411-22.

31. Deligezer U, Dalay N. Expression of the TRAIL Receptors in Blood Mononuclear Cells in leukemia. Pathol Oncol Res 2007;13:290-4.

32. Jin Z, El-Deiry W. Overview of cell death signalling pathways. Cancer Biol Ther 2005; 4:139-63.

33. Krammer PH. CD95's deadly mission in the immune system. Nature 2000;407:789-95.

34. Pfaffl MW. A new mathematical model for relative quantification in real-time RT-PCR. Nucleic Acids Res 2001;29:e45.

35. Ishii T, Bruno E, Hoffman $R$, et al. Involvement of various hematopoietic-cell lineages by the JAK2V617F mutation in polycythemia vera. Blood 2006;108:3128-34.

36. Delhommeau F, Dupont S, Tonetti C, et al. Evidence that the JAK2 G1849T (V617F) mutation occurs in a lymphomyeloid progenitor in polycythemia vera and idiopathic myelofibrosis. Blood 2007;109:71-7.

37. Pardanani A, Lasho TL, Finke C, et al. Extending Jak2V617F and MpIW515 mutation analysis to single hematopoietic colonies and B and T lymphocytes. Stem Cells 2007;25:2358-62.

38. Bogani C, Guglielmelli P, Antonioli E, et al. B-, T-, and NK-cell lineage involvement in JAK2V617F-positive patients with idiopathic myelofibrosis. Haematologica 2007;92:258-9

39. Contini $\mathbf{P}$, Zocchi MR, Pierri I, et al. In vivo apoptosis of CD8(+) lymphocytes in acute myeloid leukemia patients: involvement of soluble HLA-I and Fas ligand. Leukemia 2007;21:253-60.
40. Bárcena A, Park SW, Banapour B, et al. Expression of Fas/CD95 and Bcl-2 by primitive haematopoietic progenitors freshly isolated from human fetal liver. Blood 1996;88:2013-25.

41. De Maria R, Testa U, Luchetti L, et al. Apoptotic role of Fas/Fas ligand system in the regulation of erythropoiesis. Blood 1999;93:796-803.

42. Alenzi F0, Marley SB, Lewis JL, et al. A role for the Fas/Fas ligand apoptotic pathway in regulating myeloid progenitor cell kinetics. Exp Hematol 2002;30:1428-35.

43. Pearl-Yafe M, Yolcu ES, Stein J, et al. Expression of Fas and Fas-ligand in donor haematopoietic stem and progenitor cells is dissociated from the sensitivity to apoptosis. Exp Hematol 2007;35:1601-12.

44. Rothstein TL, Zhong X, Schram BR, et al. Receptor-specific regulation of B-cell susceptibility to Fas-mediated apoptosis and a novel Fas apoptosis inhibitory molecule. Immunol Rev 2000:176:116-33.

45. Scheneider TJ, Fischer GM, Donohoe TJ, et al. A novel gene coding for a Fas apoptosis Inhibitory Molecule (FAIM) isolated from Inducible Fas-resistant B lymphocytes. J Exp Med 1999;189:949-56.

46. Sole C, Dolcet X, Segura MF, et al. The death receptor antagonist FAIM promotes neuritis outgrowth by a mechanism that depends on ERK and NF-kappa B signalling. J Cell Biol 2004:167:479-92.

47. Segura MF, Sole C, Pascual M, et al. The long form of Fas apoptotic inhibitory molecule is expressed specifically in neurons and protects them against death receptor-triggered apoptosis. J Neurosci 2007:27:11228-41.

48. Tenedini E, Fagioli ME, Vianelli N, et al. Gene expression profiling of normal and malignant CD34-derived megakaryocytic cells. Blood 2004;104:3126-35.

49. Ashkenazi A, Pai RC, Fong $S$, et al. Safety and antitumor activity of recombinant soluble Apo2 ligand. J Clin Invest 1999;104:155-62.

50. Walczak H, Miller RE, Ariail K, et al. Tumoricidal activity of tumor necrosis factor-related apoptosis-inducing ligand in vivo. Nat Med 1999;5:157-63.

51. LeBlanc HN, Ashkenazi A. Apo2L/TRAIL and its death and decoy receptors. Cell Death Differ 2003:10:66-75.

52. Sanlioglu AD, Korcum AF, Pestereli E, et al. TRAIL death receptor-4 expression positively correlates with the tumor grade in breast cancer patients with invasive ductal carcinoma. Int J Radiat Oncol Biol Phys 2007:69:716-23.

53. Cooper WA, Kohonen-Corish MR, Zhuang L, et al. Role and prognostic significance of tumor necrosis factor-related apoptosis-inducing ligand death receptor DR5 in nonsmall-cell lung cancer and precursor lesions. Cancer 2008;113:135-42.

54. Wang $\mathbf{X}$, Chen W, Zeng W, et al. Akt-mediated eminent expression of c-FLIP and Mcl-1 confers acquired resistance to TRAIL-induced cytotoxicity to lung cancer cells. Mol Cancer Ther 2008; 7:1156-63.

55. Han J, Hou W, Goldstein LA. Involvement of protective autophagy in TRAILresistance of apoptosis defective tumor cells. J Biol Chem 2008;283:19665-77.

56. Almasan A, Ashkenazi A. Apo2L/TRAlL: apoptosis signalling, biology, and potentia for cancer therapy. Cytokine Growth Factor Rev 2003;14:337-48.

57. Passamonti F, Rumi E, Pietra D, et al. Relation between JAK2 (V617F) mutation status, granulocyte activation, and constitutive mobilization of CD34+ cells into peripheral blood in myeloproliferative disorders. Blood 2006;107:3676-82.

58. Wang W, Prince CZ, Mou Y, et al. Notch3 signaling in vascular smooth muscle cells induces c-FLIP expression via ERK/MAPK activation: resistance to Fas ligand-induced apoptosis. J Biol Chem 2002;277:21723-9.

59. Skurk C, Maatz H, Kim HS, et al. The Akt-regulated forkhead transcription factor FOXO3a Controls endothelial cell viability through modulation of the caspase-8 inhibitor FLIP. J Biol Chem 2004;279:1513-25.

60. Starck L, Scholz C, Dorken B, et al. Costimulation by CD137/4-1BB inhibits T cell apoptosis and induces Bcl-xL and c-FLIP(short) via phosphatidylinositol 3-kinase and AKT/protein kinase B. Eur J Immunol 2005:35:1257-66.

61. Wang MJ, Liu S, Liu Y, et al. Actinomycin D enhances TRAlL-induced caspase-dependent and independent apoptosis in SH-SY5Y neuroblastoma cells. Neurosci Res 2007:59:40-6.

62. Huo J, Xu S, Guo K, et al. Genetic deletion of faim reveals its role in modulating c-FLIP expression during CD95-mediated apoptosis of lymphocytes and hepatocytes. Cell Death Differ 2009;16:1062-70. 